\title{
A comparison of short-stem prostheses and conventional stem prostheses in primary total hip arthroplasty: a systematic review and meta-analysis of randomized controlled trials
}

\author{
Zhen Zhang, Qiqi Xing, Jingyi Li, Zichao Jiang, Yixiao Pan, Yihe Hu, Long Wang \\ Department of Orthopedics, Xiangya Hospital, Central South University, Changsha, China \\ Contributions: (I) Conception and design: Z Zhang, L Wang; (II) Administrative support: L Wang, Y Hu; (III) Provision of study materials or patients: \\ All authors; (IV) Collection and assembly of data: Z Zhang, J Li, Z Jiang, Y Pan; (V) Data analysis and interpretation: Z Zhang, L Wang, Q Xing; (VI) \\ Manuscript writing: All authors; (VII) Final approval of manuscript: All authors. \\ Correspondence to: Long Wang. Department of Orthopedics, Xiangya Hospital, Central South University, No. 87 Xiangya Road, Changsha 410008, \\ China. Email: dr_wanglong@csu.edu.cn.
}

\begin{abstract}
Background: In total hip arthroplasty (THA), short-stem prostheses (SS) were designed to achieve better preservation of proximal femoral bone stock and stability than conventional stem prostheses (CS), however these effects are controversial. We aimed perform a systematic review and meta-analysis to evaluate the effectiveness of SS and CS in primary THA.

Methods: Relevant randomized controlled trials (RCTs) involving the comparison of SS and CS in primary THA were screened using the electronic databases PubMed, Embase and Web of Science. Data were analyzed with the RevMan 5.3 software program and evaluated with mean difference (MD), risk ratio (RR) and $95 \%$ confidence intervals (CIs) by random or fixed-effect models.

Results: Sixteen RCTs involving 1,233 patients (1,486 hips) were included. Compared with CS, the incidence of thigh pain was significantly reduced with Proxima SS (RR 0.13, 95\% CI, 0.03-0.51; P=0.004). Bone mineral density (BMD) with femoral neck-preserved SS [SS (I)] showed less decrease in Gruen zone 1 (MD 14.60, 95\% CI, 10.67-18.54; P<0.00001) and Gruen zone 7 (MD 9.72, 95\% CI, 5.21-14.23; P<0.0001) than CS. However, the changes of BMD were not significantly different between the SS without femoral neck preservation group [SS (II)] and the CS group. In addition, no significant differences were found in the revision rate, Harris Hip Score (HHS), or maximum total point motion (MTPM) between the SS and CS groups.

Conclusions: The results of this study showed that compared with CS, Proxima SS decreased the incidence of thigh pain and that SS (I) provided better proximal bone remodeling than CS. But the revision rates, HHS, and MTPM between SS and CS were similar. However, the findings of this meta-analysis require further verification in high-quality RCTs.
\end{abstract}

Keywords: Total hip arthroplasty (THA); short-stem prostheses (SS); conventional stem prostheses (CS); metaanalysis

Submitted May 20, 2020. Accepted for publication Oct 23, 2020.

doi: 10.21037/atm-20-4043

View this article at: http://dx.doi.org/10.21037/atm-20-4043 


\section{Introduction}

Total hip arthroplasty (THA) is still one of the most effective treatments for end-stage hip diseases (1). THA has been widely used in the past few decades. More than 193,000 THA operations are performed annually in the United States, and the number is expected to increase $170 \%$ by 2030 . Statistically, adults aged $35-50$ years old account for approximately $10 \%$ of THA operations annually (2). Increased surgical volume and younger patients will increase the burden of revision surgery (3). Although THA has achieved great success, complications such as aseptic loosening, periprosthetic joint infection, instability, leglength discrepancy and periprosthetic femoral fracture still occur sometimes. In addition, stress shielding and thigh pain may occur after surgery $(4,5)$; stress shielding can cause bone loss around the prostheses, which can lead to periprosthetic fractures in the area of bone defects (6).

Data from short-term and long-term follow-up studies indicated that thigh pain is a serious postoperative complication and the incidence of recalcitrant thigh pain with cementless designs ranges from $0.5 \%$ to $40 \%(7,8)$. In most cases, thigh pain is mild to moderate and known as an intermittent and self-limiting phenomenon, which does not necessitate medication (9); however, it is a main factor of dissatisfaction after THA, the incidence is also increasing with the increase in the use of cementless stems. If thigh pain does not disappear 1 to 2 years after THA and the activity is severely restricted, surgery should be considered (8). So, regarding the current situation, thigh pain is worth discussing.

In general, two types of prostheses are available in primary THA: conventional stem prostheses (CS) and short-stem prostheses (SS). In recent years, the SS which preserving the femoral neck have attracted more attention, primarily for their bone-protective properties provide favorable conditions for revision, as well as biomechanical advantages. In addition, neck-sparing stem design can facilitate minimally invasive surgical techniques (10). The standard lengths of CS are usually $120-150 \mathrm{~mm}$ (11), while the lengths of SS are usually $<120 \mathrm{~mm}(12,13)$. SS are designed to preserve the proximal femoral bone stock and get the primary stability by a metaphyseal fixation, prevent the distal medullary cavity from being invaded and reduce stress shielding $(14,15)$.

Some new clinical studies (16-19) have been published since the publication of the last relevant meta-analysis (20). One of these studies (19) showed that thigh pain after THA is higher with SS than CS, which is inconsistent with the result of a previous meta-analysis by Huo et al. (21). In the meta-analysis by Liang et al. (20), the evaluation data of BMD showed high heterogeneity $\left(\mathrm{I}^{2}=85 \%\right)$; similarly high heterogeneity was found for the subgroup data $\left(\mathrm{I}^{2}=85 \%\right.$, $\left.\mathrm{I}^{2}=89 \%\right)$. Analysis of MTPM data also showed high heterogeneity within each subgroup $\left(I^{2}=56 \%, I^{2}=72 \%\right)(20)$, which must be resolved by adding new data or performing a new subgroup analysis. The conflicting findings reported in new study, the need to update the current data, and the high heterogeneity in the current literature have motivated us to perform a new meta-analysis (22). To improve the statistics and analysis of outcomes regarding thigh pain, bone remodeling, prosthesis survival rate, MTPM and functional results after primary THA, we conducted an updated systematic review and meta-analysis using all currently available RCTs to assess the effectiveness of SS and CS in primary THA.

We present the following article in accordance with the PRISMA reporting checklist (available at http://dx.doi. org/10.21037/atm-20-4043).

\section{Methods}

This meta-analysis was designed and conducted according to the preferred reporting items for systematic review and meta-analysis protocols (PRISMA-P) 2015 statement (23).

\section{Search strategy}

Our study carried out a systematic literature search of all relevant RCTs involving SS and CS in primary THA before November 2019 in PubMed, Embase and Web of Science. We screened databases by using the following keywords and their combinations: short stem, conventional stem, hip arthroplasty, hip replacement, randomized controlled trial (RCT), and controlled clinical trial. Reviewers browsed the references of the included studies to identify potential eligible studies. The Cochrane Handbook was used to assess the quality of all included studies to ensure that our metaanalysis was reliable and verifiable.

\section{Inclusion and exclusion criteria}

The selection of studies for inclusion was independently performed by two reviewers and included screening of titles and abstracts and assessing full texts for potential eligible studies. The two investigators resolve objections through 
discussion or consultation with other investigators. The inclusion criteria for this meta-analysis were as follows: (I) prospective randomized controlled clinical studies; (II) patients with hip diseases such as osteoarthritis, femoral head necrosis, traumatic arthritis or femoral neck fracture assigned to undergo primary cementless THA; (III) studies comparing SS and CS in primary THA; and (IV) an unlimited follow-up time and patient age. The exclusion criteria were as follows: (I) nonprospective randomized controlled clinical studies; (II) nonhuman subjects; and (III) revision surgery.

\section{Data extraction and quality assessment}

Two investigators independently extracted relevant data from the included studies. Including the first author's name, year of publication, number of patients, age, sex ratio, BMI, follow-up duration, prosthesis type and outcome measurements. Any uncertainty or disagreement was discussed by the two investigators or resolved by consensus through consultation with other investigators. We contacted the authors of the included RCTs as necessary to obtain any missing data. The Cochrane Collaboration tool (24) was used to evaluate the methodological quality of each study by the risk of bias, including randomization, allocation of concealment, blinding methods, selective reporting, population similarity at baseline, incomplete results data, etc. We used funnel plot to evaluate publication bias and screened studies from three electronic databases (PubMed, Embase and Web of Science) to decrease the location bias.

\section{Outcome measurements}

The results evaluated in our meta-analysis include the incidence of thigh pain; dual-energy X-ray absorptiometry (DEXA) measures of bone mineral density (BMD) changes in different Gruen zones to assess bone remodeling; the Harris Hip Score (HHS) to evaluate functional results; revision rates to assess prosthetic survival; and the maximum total point motion (MTPM) to assess prosthetic migration.

\section{Statistical analysis and data synthesis}

The meta-analysis was performed with RevMan 5.3. Considering the characteristics of the data, continuous variables were expressed as the mean difference (MD) with $95 \%$ confidence intervals (CIs), while binary variables were expressed as risk ratios (RRs). $\mathrm{I}^{2}$ was used to evaluate heterogeneity. A random effect model was used when statistical heterogeneity existed $\left(\mathrm{I}^{2} \geq 50 \%\right)$; otherwise, a fixed effect model was used. If $\mathrm{I}^{2} \geq 50 \%$, to detect the impact of each data set on the overall effects of the analyses, a sensitivity analysis was conducted to assess the stability of the results and access potential sources of heterogeneity by sequentially deleting a single study involved in the metaanalysis. Subgroup analyses were performed according to different follow-up years and different types of prostheses to verify the source of heterogeneity. $\mathrm{P}<0.05$ was considered statistically significant.

\section{Results}

\section{Description of the included studies}

Our initial electronic search yielded 2,050 related studies, of which 399 were removed due to being duplicates. We identified 50 articles after reading the title and abstract, of which 34 articles were excluded after reading the full text. Finally, 16 RCTs (16-19,25-36) published from 2006 to 2019 were included in this meta-analysis; they involved 9 different types of SS and 1,233 patients (Figure 1). The characteristics of the eligible studies are shown in Table 1. The sample size was 731 hips in the SS group and 755 hips in the CS group, and the population size ranged from 21 to 200 patients. The follow-up time ranged from 6 weeks to 11.8 years (average 4.0 years).

\section{Risk of bias of the included studies}

All 16 included studies were RCTs, of which two were multicenter and two were double-blind RCTs. A clear generation of random sequences and allocation concealment were employed by 11 studies, indicating a low risk of bias, while the remaining five trials $(17,29-32)$ did not report their sequence generation, and three trials $(17,32,34)$ did not mention allocation concealment. The blinding of participants and personnel was unclear in four studies $(17,31,32,34)$, and a high risk of bias was found in one study (30) in which patients were not blinded. Blinding of outcome assessment was unclear in four studies $(17,30,32,35)$, and a high risk of bias was observed in one study (25) in which researchers and surgeons evaluated the clinical and imaging results. All of the included articles displayed a low risk of bias for incomplete outcomes, selective outcome reporting, and other biases. Details of the bias assessment are shown in Figure 2. The funnel polts 


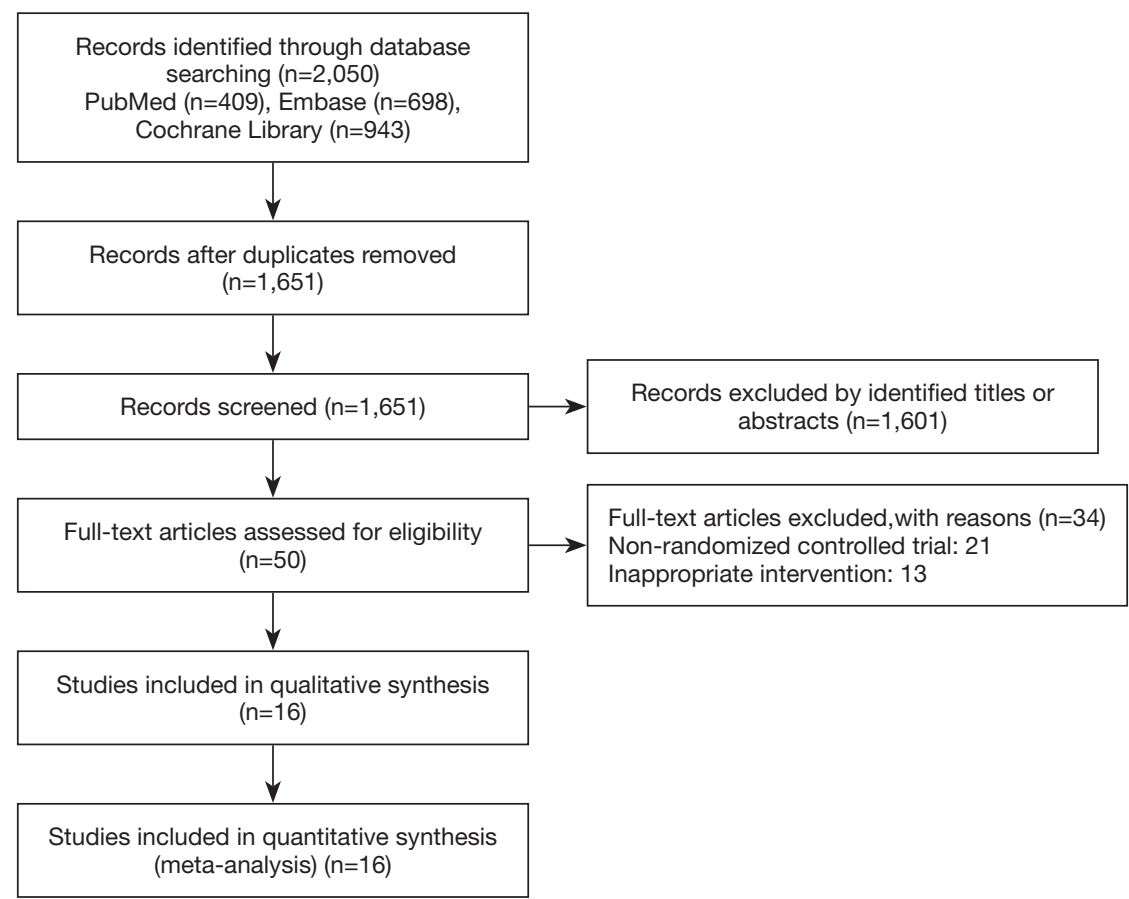

Figure 1 Flow diagram of the study selection process.

Table 1 Overview of the characteristics of the included studies

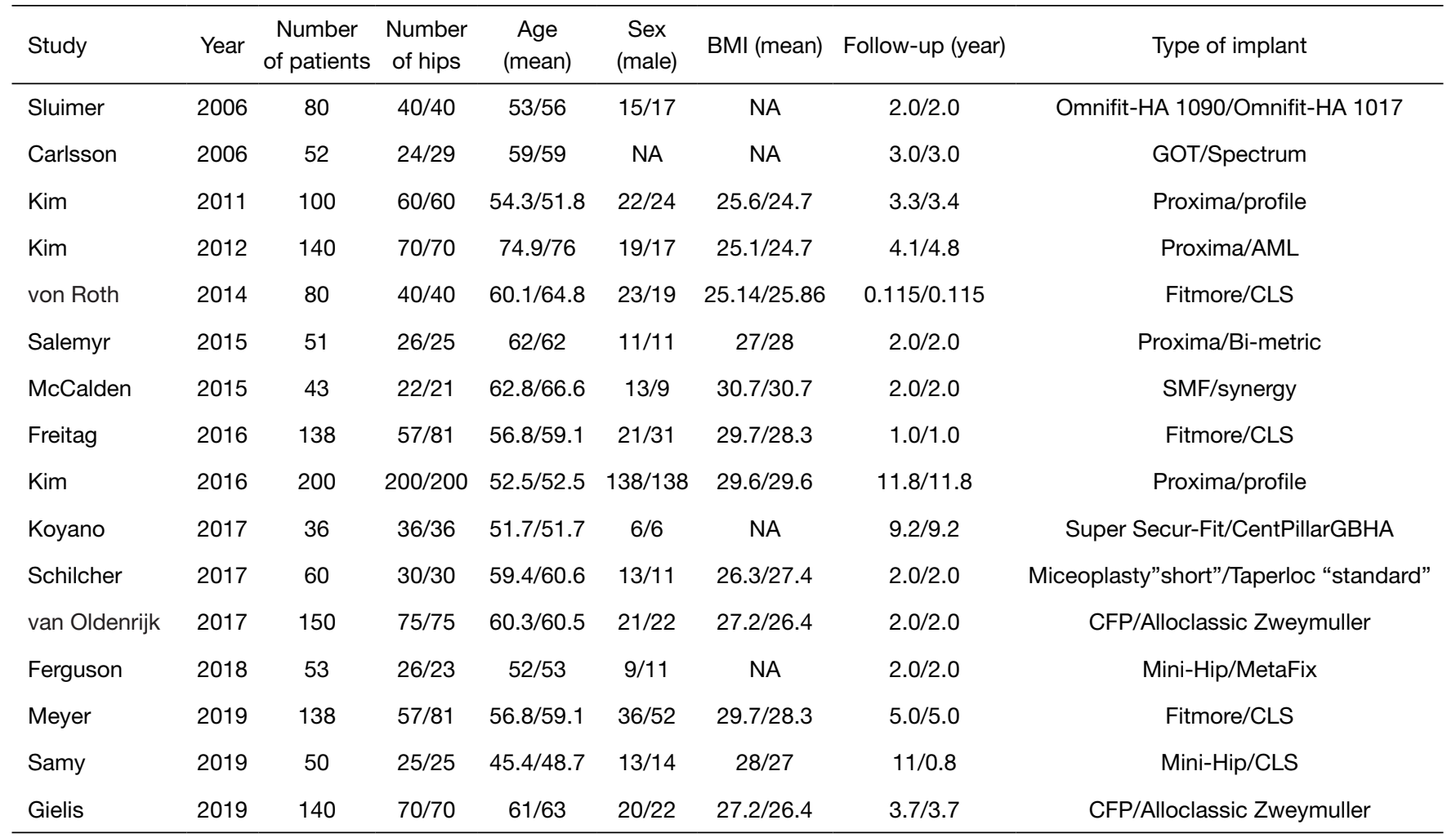




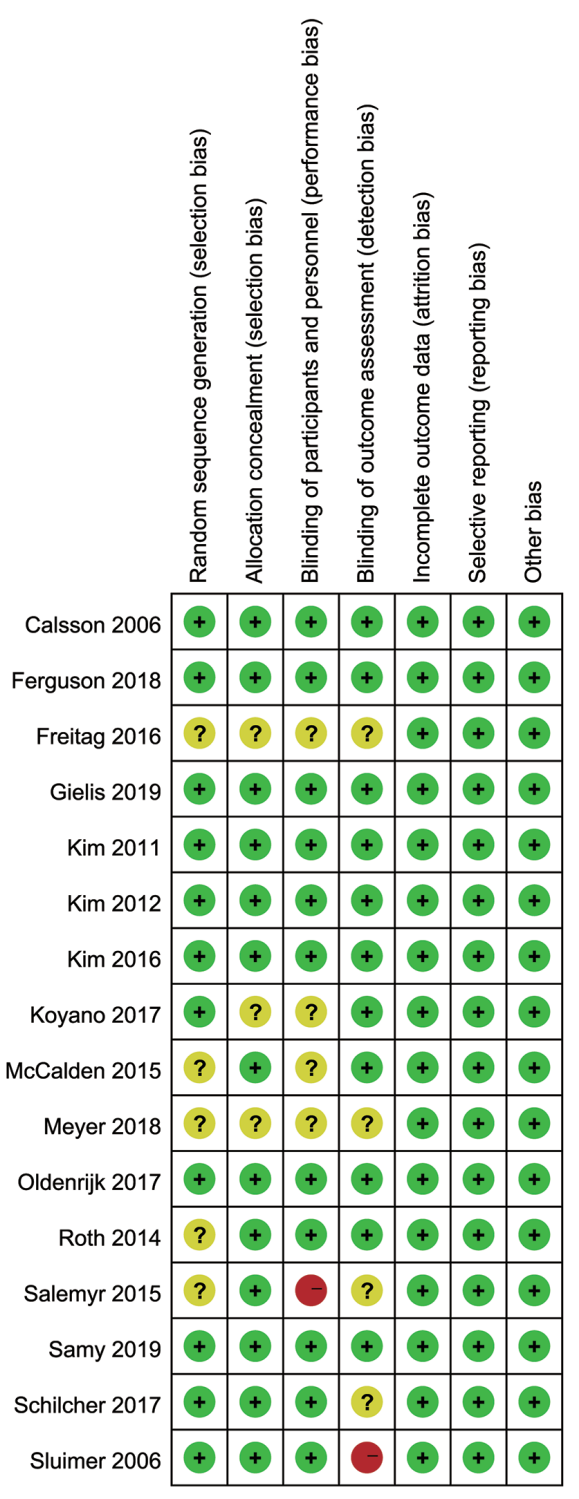

Figure 2 Risk of bias summary for each included study.

of thigh pain, BMD, revision rate, HHS and MTPM all show a sharp head and a big bottom, which indicated low publication bias (Figures S1-S6).

\section{Outcomes of the meta-analyses}

\section{Thigh pain}

Five studies reported thigh pain, three of which used a 10 -point VAS to score thigh pain. The first study (27) reported that $5(10 \%)$ patients in the CS group had mild or moderate thigh pain and no patient in the SS group had thigh pain at the last follow-up. The second study (28) reported that none of the patients with SS had thigh pain but that 11 patients $(16 \%)$ with CS had thigh pain. The third study (33) reported that 26 patients (13\%) had mild thigh pain, 4 patients $(2 \%)$ experienced moderate thigh pain, and 1 patient $(0.5 \%)$ suffered severe thigh pain in the CS group, while 2 patients $(1 \%)$ experienced severe thigh pain in the SS group. Salemyr et al. (30) reported that 3 patients in the CS group experienced thigh pain, and 2 patients in the SS group suffered thigh pain. However, Gielis et al. (19) reported that thigh pain occurred in 15 patients in the CS group and 24 patients in the SS group (Figure 3).

Although the methods to quantify thigh pain are different, we can enumerate the total number of patients who complained of pain, totaling 28 of 426 patients in the SS group and 65 of 425 patients in the CS group. No significant difference was found in thigh pain between the two groups (RR $0.23,95 \% \mathrm{CI}, 0.03-1.60 ; \mathrm{P}=0.14 ; \mathrm{I}^{2}=87 \%$ ) (Figure $3 A$ ), and the heterogeneity was high. Thus, a sensitivity analysis was conducted to further analyze the sources of heterogeneity.

\section{BMD}

Ten studies, including 372 hips, reported BMD in different Gruen zones. BMD was measured by DEXA in these studies. Data on BMD changes between 1 week and 1-2 years after surgery were available for seven studies $(17,18,25,30,32,34,35)$. BMD decreased immediately in all Gruen zones in both the SS and CS groups after surgery; however, compared to that in other Gruen zones, the BMD in Gruen 1 (G1) and Gruen 7 (G7) decreased significantly by the two-year follow-up (Figures 4 and 5). Studies grouped by follow-up time alone led to high statistical heterogeneity (Figure $4 A$ and $5 A$ ). In five included studies, two studies $(18,30)$ mentioned that the Mini-Hip stem (Corin) and Proxima stem (DePuy) retained the femoral neck during osteotomy, whereas three other studies $(25,32,35)$ showed that the Omnifit-HA 1090 stem (Osteinics), Fitmore stem (Zimmer), and Miceoplasty "short" stem (Biomet) did not retain the femoral neck. Our study divided the SS into a femoral neck-preserving prostheses group [SS (I)] and a non-preserving femoral neck prostheses group [SS (II)] for discussion.

In G1, compared with CS, SS (I) decreased the reduction in BMD significantly at 1 year (MD 13.76, 95\% CI, 8.5318.99; $\mathrm{P}<0.00001)$ and 2 years after surgery (MD 15.71, 95\% CI, 9.73-21.68; $\mathrm{P}<0.00001)$ and overall (MD 14.60, 


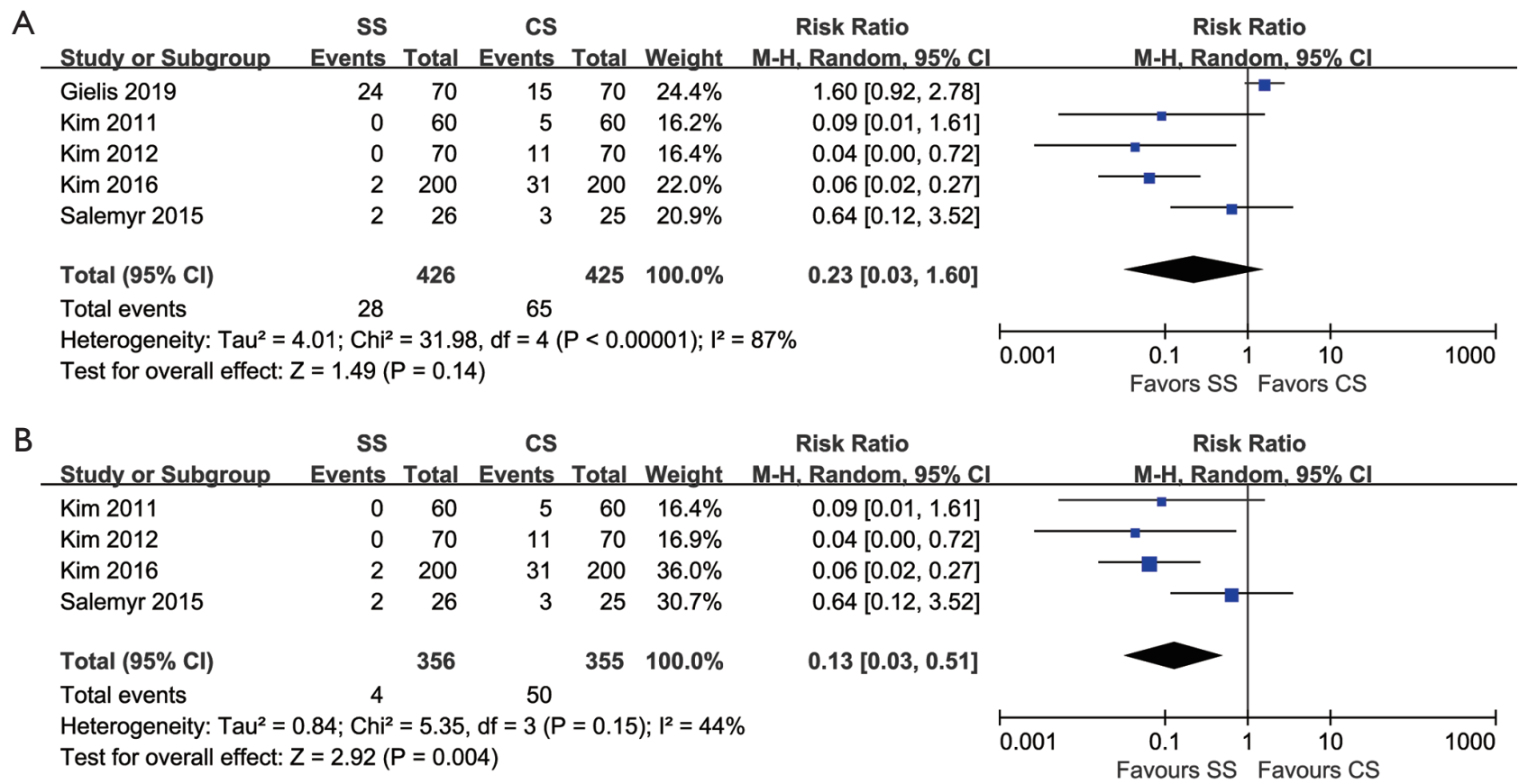

Figure 3 Forest plot for thigh pain. (A) Including Geilis' RCT; (B) Excluding Geilis' RCT.

95\% CI, 10.67-18.54; $\mathrm{P}<0.00001 ; \mathrm{I}^{2}=0 \%$; Figure $\left.4 B\right)$ In G7, compared with CS, SS (I) significantly decreased the reduction in BMD at 1 year after surgery (MD 10.77, 95\% CI, 5.18-16.37; $\mathrm{P}=0.0002$ ), but no significant difference was found at the 2-year follow-up (MD 8.84, 95\% CI, -0.50-18.18; $\mathrm{P}=0.06)$; however, collectively, these results were significant (MD 9.72, 95\% CI, 5.21-14.23; P<0.0001; $\mathrm{I}^{2}=32 \%$; Figure 5 B). Compared with CS, SS (II) showed no difference in G1 (MD $-1.96,95 \% \mathrm{CI},-4.99$ to 0.97 ; $\mathrm{P}=0.19 ; \mathrm{I}^{2}=23 \%$ ) or $\mathrm{G} 7$ (MD 4.03, 95\% CI, -0.39 to 8.44 ; $\mathrm{P}=0.07 ; \mathrm{I}^{2}=39 \%$; Figures 4 C, 5 C).

\section{Revision rates}

Thirteen studies reported revision rates with 30 revisions in 1,406 hips. The main reasons for revision were aseptic loosening, periprosthesis fractures and dislocations. We divided the data into three groups depending on the time that revision occurred $(<2,2-8$, and $>8$ years) and found no heterogeneity between subgroups $\left(\mathrm{I}^{2}=0 \%\right)$. The results for every subgroup ( $<2$ years: RR 1.28 ; $95 \%$ CI, $0.50-3.28$; $\mathrm{P}=0.60 ; 2-8$ years: RR 1.36; 95\% CI, 0.48-3.84; $\mathrm{P}=0.56$; $>8$ years: RR 3.00; 95\% CI, 0.33-27.5; $\mathrm{P}=0.33)$ and overall (RR 1.43; 95\% CI, 0.74-2.77; $\mathrm{P}=0.28$ ) (Figure 6) did not reveal significant differences between the SS and CS groups.

\section{HHS}

Eleven studies including 1,280 hips reported HHS. No significant difference was observed between the two groups at the end of follow-up (MD $-0.32,95 \% \mathrm{CI},-0.94$ to 0.31 ; $\mathrm{P}=0.32 ; \mathrm{I}^{2}=0 \%$; Figure 7 ).

\section{Maximum total point motion (MTPM)}

MTPM was used to evaluate femoral migration in five studies with 210 hips in the SS group and 208 hips in the CS group. No significant differences were found between the SS group and CS group at the 1-year (MD 0.02; 95\% CI, $-0.18-0.22 ; \mathrm{P}=0.84 ; \mathrm{I}^{2}=33 \%$ ) or 2-year follow-ups (MD $0.08,95 \% \mathrm{CI},-0.16-0.31 ; \mathrm{P}=0.53 ; \mathrm{I}^{2}=57 \%$ ) or overall (MD $0.01 ; 95 \%$ CI, $-0.07-0.08 ; \mathrm{P}=0.83 ; \mathrm{I}^{2}=42 \%$; Figure 8 ).

\section{Sensitivity analysis}

Thigh pain and MTPM were still highly heterogeneous after subgroup analysis. Thus sensitivity analysis was performed by deleting each study separately to identify potential sources of heterogeneity.

The results of the heterogeneity analysis and $\mathrm{P}$ value changed for thigh pain only when Gielis et al. (19) was deleted. It is worth noting that Collum Femoris Preserving (CFP) short-stem prostheses were used only in Gielis 


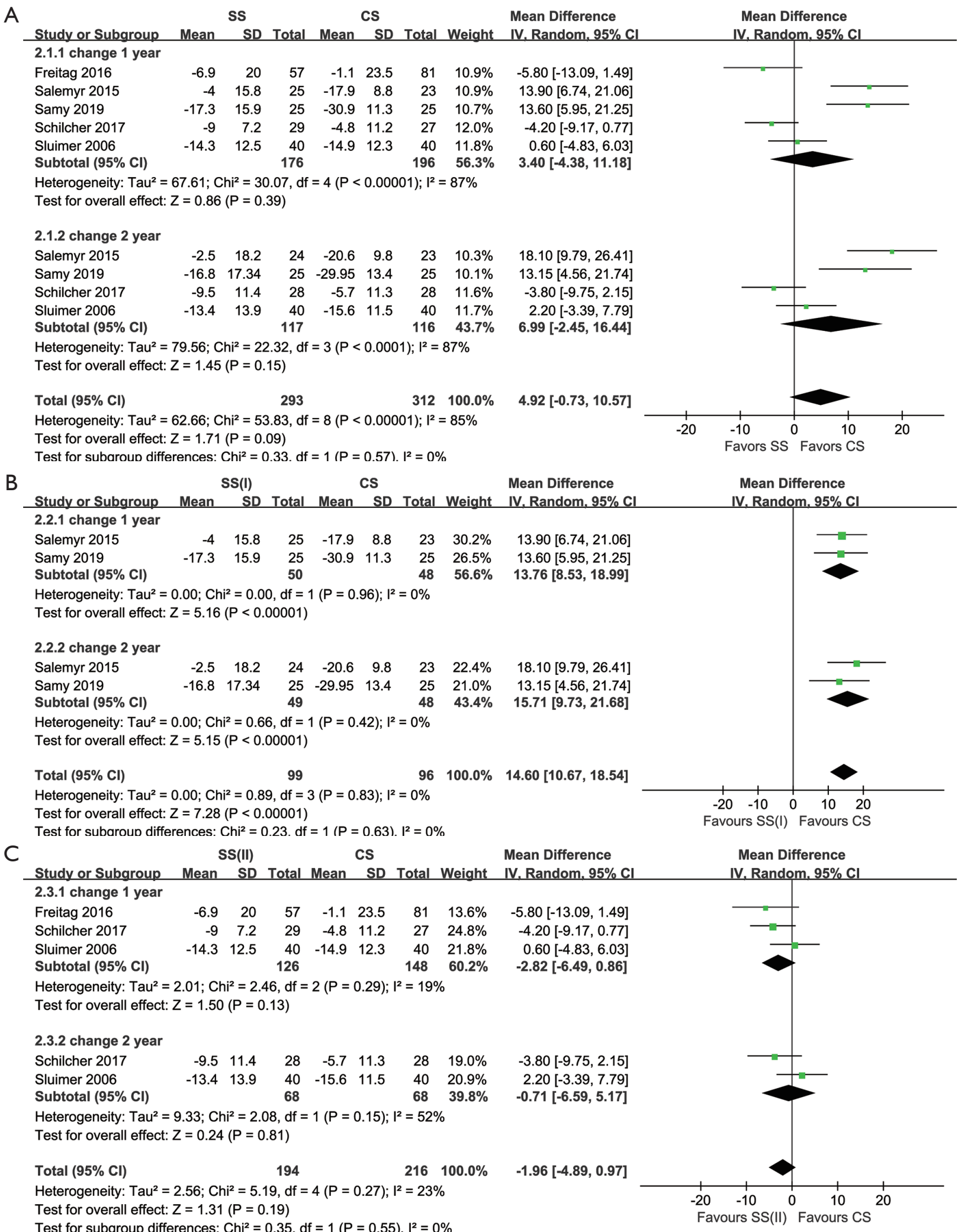

Figure 4 Forest plot for BMD changes in Gruen zone 1. (A) Including all types of SS; (B) Including SS (I); and (C) Including SS (II). 


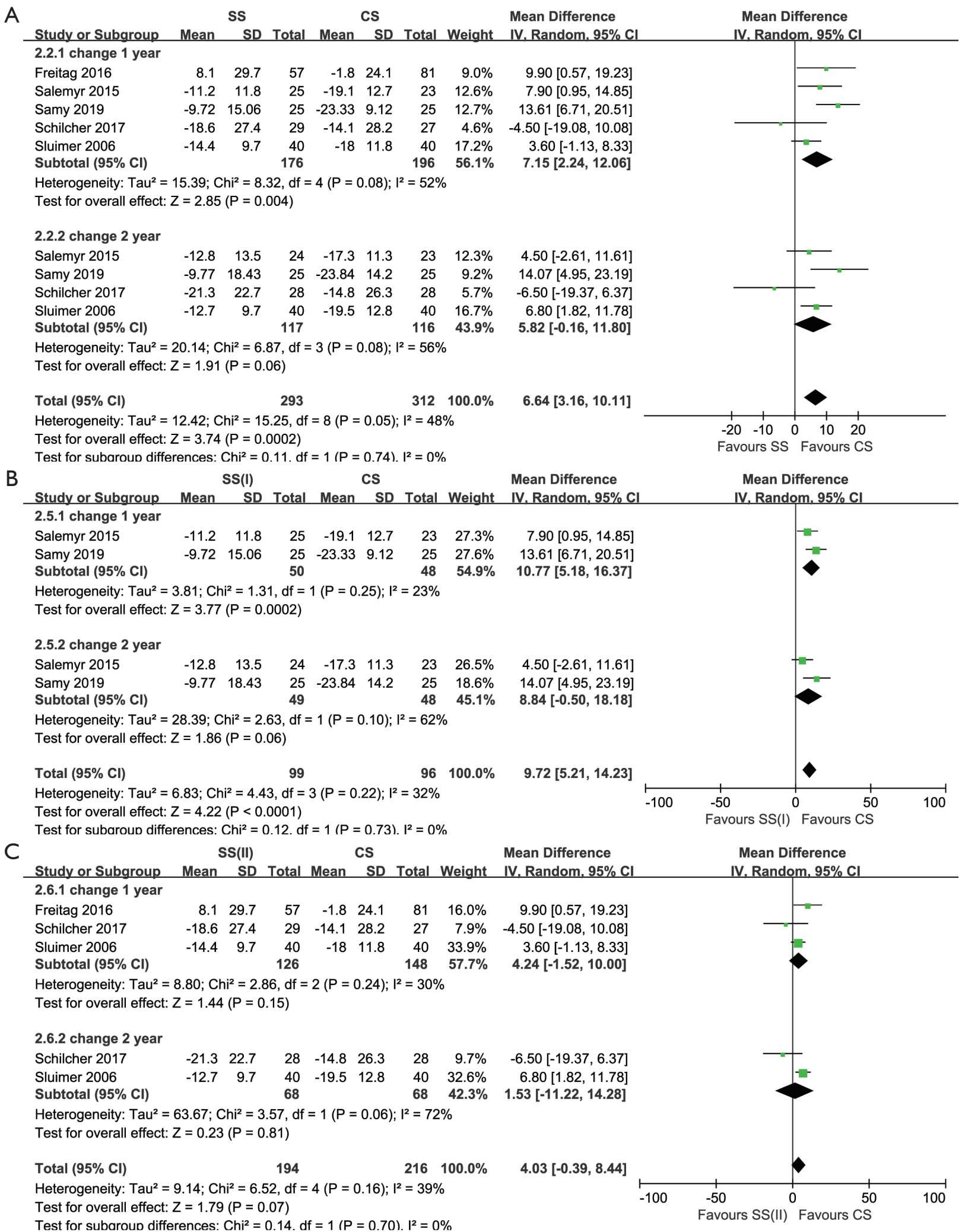

Figure 5 Forest plot for BMD changes in Gruen zone 7. (A) Including all types of SS; (B) Including SS (I); and (C) Including SS (II). 


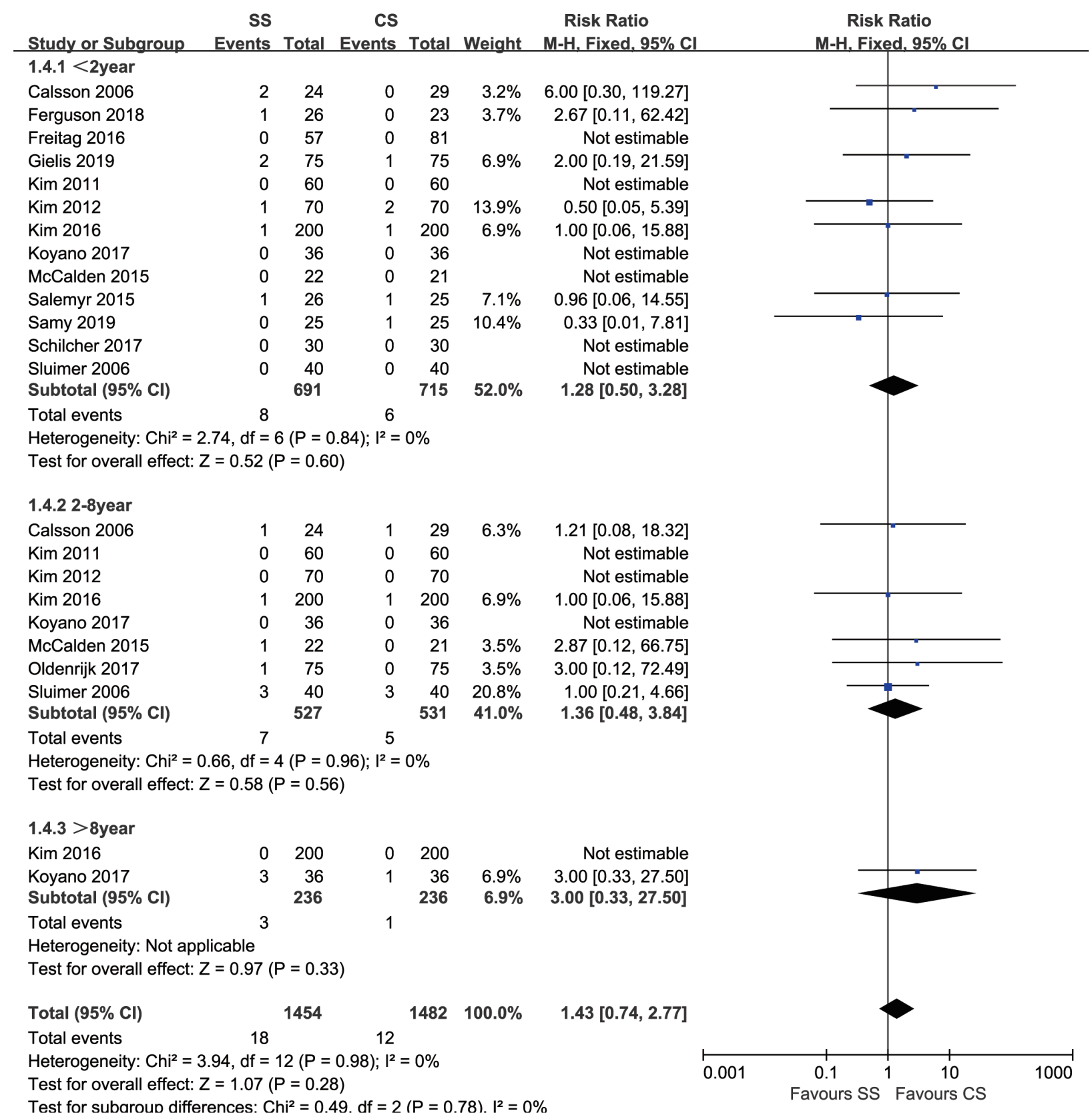

Figure 6 Forest plot for revision rates.

et al. (19), while Proxima (DePuy) short-stem prostheses were used in the other four studies. The data analysis of these four studies indicated that the incidence of thigh pain in the Proxima SS group was significantly less than that in the CS group (RR 0.13, 95\% CI, 0.03-0.51; $\mathrm{P}=0.004$; Figure 3B).

The results of the heterogeneity analysis changed for MTPM when Salemyr et al. (30) was removed. The P value was always greater than 0.05 as heterogeneity decreased $\left(\mathrm{I}^{2}=0 \%\right)$. There was no significant difference in MTPM between the SS and CS groups, which is the same as our previous conclusion.

\section{Discussion}

This meta-analysis included 16 RCTs involving 1,233 


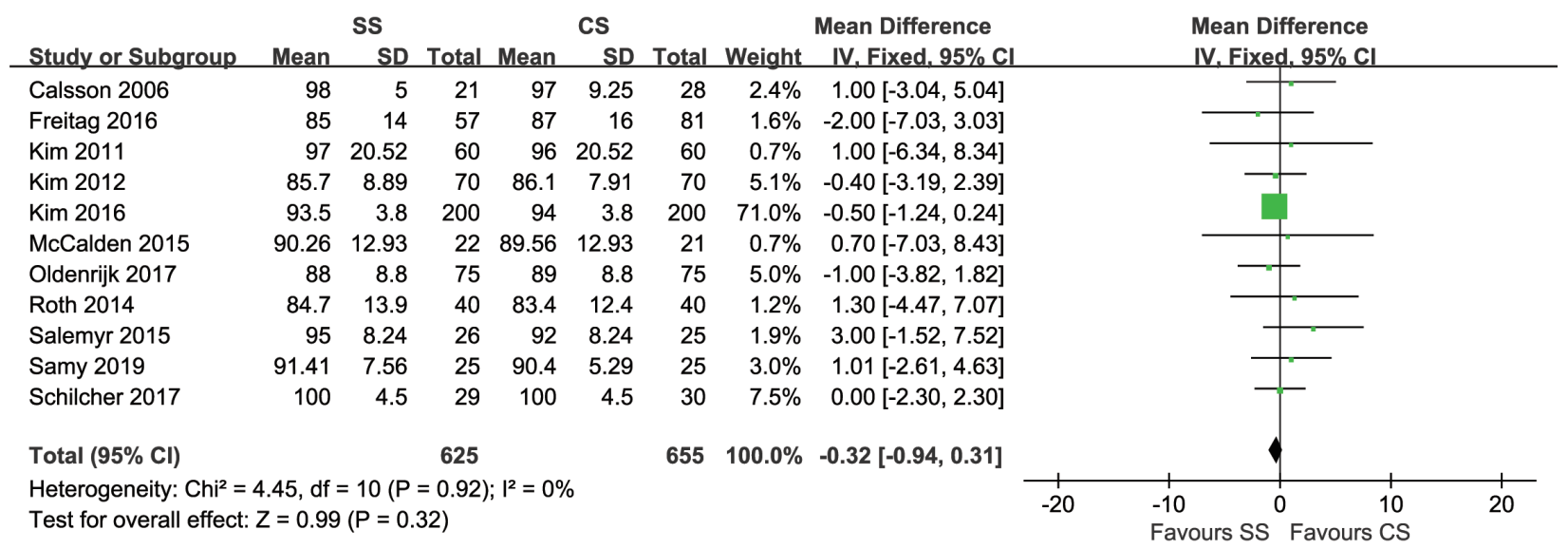

Figure 7 Forest plot for Harris Hip Scores.

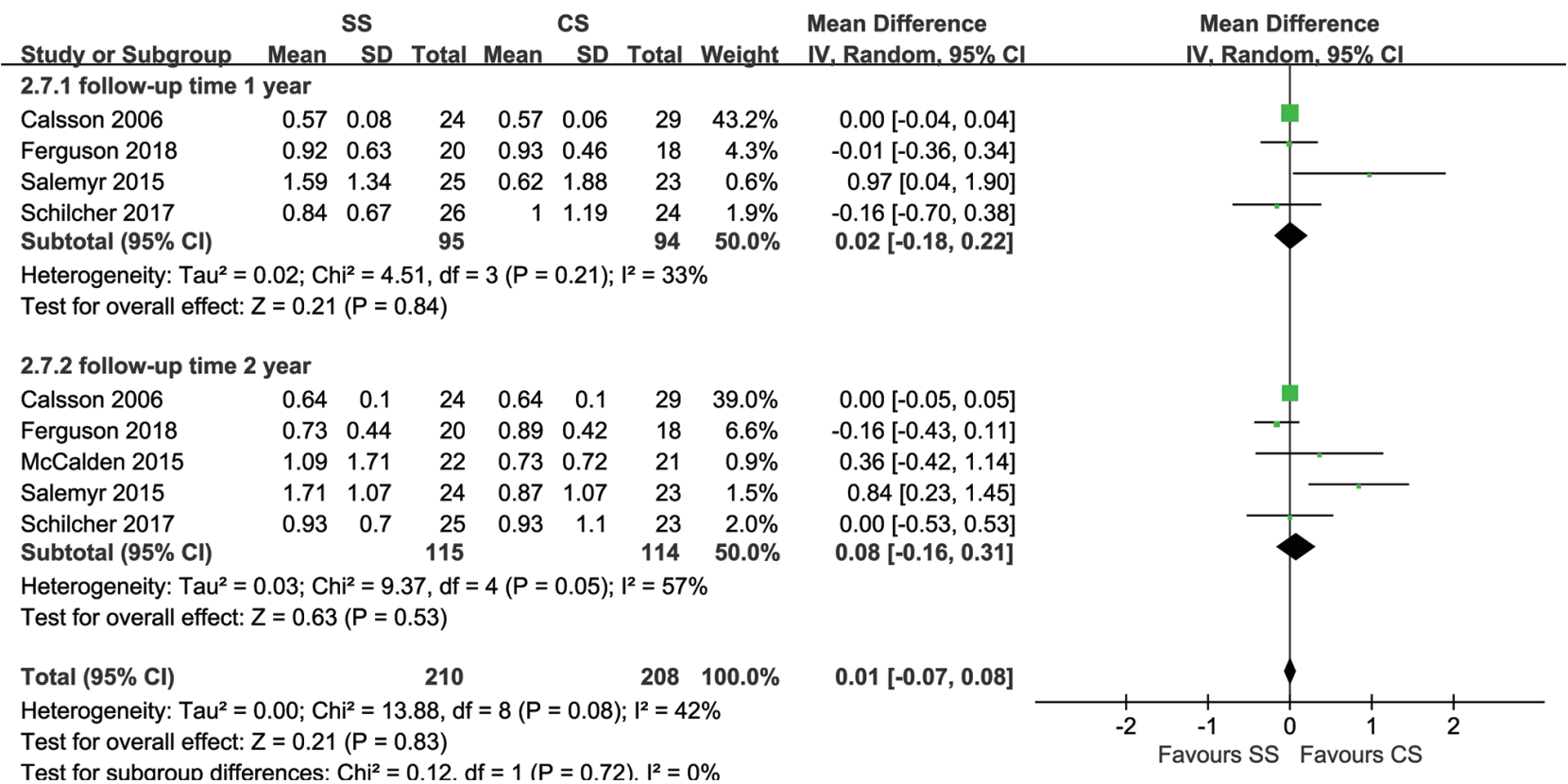

Figure 8 Forest plot for maximum total point motion.

patients, and evaluated the effectiveness and safety of SS and CS. The results showed that compared to CS, SS (I) effectively reduced BMD changes in G1 and G7, but there was no significant difference for SS (II). No significant differences were found in terms of the revision rate, HHS, or MTPM between the two groups. Due to the high heterogeneity of the thigh pain results, it was temporarily unclear whether there was a significant difference between the two groups.

Several previous meta-analyses evaluated the effectiveness of SS in primary THA. Yan et al. (37) reported that SS with a lateral flare was superior to CS in bone remodeling in G1 and G7 (prosthesis survival, functional outcomes, and migration in primary THA but SS achieved better bone remodeling than CS. However, some new RCTs have been published and were included in our study. In this metaanalysis, we tried to address whether SS can provide better bone remodeling, lower thigh pain rates, lower revision rates, smaller prosthetic migration and better clinical outcomes than CS in primary THA. 
A previous meta-analysis (21) found that SS could reduce the incidence of postoperative thigh pain; however, that analysis involved only three studies $(27,28,30)$ with a small number of subjects (SS/CS: 156/155 hips). Seven studies included in our meta-analysis mentioned thigh pain, referring particularly to postoperative complications caused by stress shielding and micromotion of the prostheses in the middle of the thigh after THA. However, pain was described in the groin or hip in two studies, which were excluded when analyzing the results for thigh pain. Ultimately, five studies $(19,27,28,30,33)$ were included (SS/CS: 426/425), with large heterogeneity observed among the data. The sensitivity analysis showed that the heterogeneity originated from Gielis et al. (19). The other studies all used Proxima short-stem prostheses, which are anatomically wedgeshaped, titanium alloys (Ti-6Al-4V) fully porous-coated with sintered beads, with a mean pore size of $250 \mu \mathrm{m}$, and they are covered with 30- $\mu \mathrm{m}$ highly amorphous hydroxyapatite (30). The design of the prostheses is characterized by a long medial stalk, a lateral flare and retention of the femoral neck $(27,33)$. The lateral flare allows the implant to rest laterally where the arciform fibers end on the greater trochanter (27), plus the fixation of the prostheses in the isthmus enables the prostheses to obtain higher stability $(27,38)$. The reasons for the low incidence of thigh pain in this type of SS may be (I) axial stability and (II) the lack of contact between the stem tip and the cortex (39).

The CFP short-stem prosthesis used by Gielis et al. is the second prosthesis after Pipino's biodynamic prosthesis that retains the femoral neck (36). CFP is characterized by a removable neck collar, retention of the femoral neck and longitudinal ribs to provide rotational stability $(19,40)$. The incidence of thigh pain in seven studies with 599 hips was $2 \%$ (39), but the rate in the study by Gielis et al. (19) was $34 \%$, which was much higher than reported in previous studies. A previous study (41) reported that the incidence of postoperative thigh pain in THA using CFP increased with a decrease in the neck protection ratio. This result suggested that the high incidence of thigh pain in CFP stems was related to the surgeon's improper surgical choice of osteotomy plane. It is hoped that future RCTs will have a clearer description of the thigh pain location, a unified assessment tool for thigh pain, and inclusion of the number of people with different pain levels to facilitate statistical analysis.

In terms of BMD changes, our results indicated that $\mathrm{SS}$ (I) could yield better bone remodeling than CS. This may be because SS (I) retains more bone mass and maintains the normal femoral alignment and the stress distribution is consistent with physiological conditions; altogether, these factors help to achieve a good biomechanical load in order to reduce stress shielding. Our meta-analysis results revealed that SS (I) and SS (II) exert different effects on bone remodeling. Current thinking indicates that the ability to increase BMD and perform bone remodeling is different for different ages and sexes (42), which may affect the conclusions of this meta-analysis. However, based on the limited data from the currently available studies, we were unable to perform subgroup analyses of the above factors.

In terms of revision rate, the reasons for revision were aseptic loosening, recurrent dislocations, and infection, periprosthetic fractures. The results of the meta-analysis showed that SS and CS have similar revision rates in the early stages after primary THA. Compared with the preoperative HHS, HHS was increased after surgery in both the SS and CS groups in 11 of the included studies, and the increase between SS and CS was not significantly different, which was consistent with previous meta-analyses and RCTs. There were five studies involving MTPM to assess the migration of prostheses, and the results indicated that there was no significant difference between SS and CS in terms of prosthesis migration.

This study was based on several RCTs from various populations to evaluated proximal bone remodeling, revision rate, HHS, and MTPM, between SS and CS. We note several limitations of our study. First, the designs of SS are various in the included studies, due to the limited number of published studies; we cannot perform subgroup analysis for each type. Second, the heterogeneities existed in sample size and followup time, and some studies used moderate quality methodology may all affect the result of meta-analysis. Third, due to the limitations of the currently available data, we cannot perform subgroup analysis on the severity of thigh pain and further discussion on confounding variables for revision rate. Fourth, our study also did not compare such as caliber index or Dorr classification. Finally, there are many confounding factors, such as preoperative femoral morphology, patient age, gender and BMI, which all affect the results, which require more high quality RCTs to further clarify.

\section{Conclusions}

Compared with CS, SS showed similar prostheses survival rates, prostheses migration and functional results in primary THA. Proxima short-stem prostheses significantly reduced the incidence of thigh pain. Femoral neck-preserved SS 
achieved better bone remodeling and saved more bone mass than CS, which benefits young patients undergoing revision surgery.

\section{Acknowledgments}

Funding: This work was supported by the National Natural Science Foundation of China (Grant No: 81601883, 81873988), and Natural Science Foundation of Hunan Province (CN) (Grant No: 2018JJ3861). We thank American Journal Experts (www.aje.com), for editing the English text of a draft of this manuscript.

\section{Footnote}

Reporting Checklist: The authors have completed the PRISMA reporting checklist. Available at http://dx.doi. org/10.21037/atm-20-4043

Data Sharing Statement: Available at http://dx.doi. org/10.21037/atm-20-4043

Peer Review File: Available at http://dx.doi.org/10.21037/ atm-20-4043

Conflicts of Interest: All authors have completed the ICMJE uniform disclosure form (available at http://dx.doi. org/10.21037/atm-20-4043). The authors have no conflicts of interest to declare.

Ethical statement: The authors are accountable for all aspects of the work in ensuring that questions related to the accuracy or integrity of any part of the work are appropriately investigated and resolved.

Open Access Statement: This is an Open Access article distributed in accordance with the Creative Commons Attribution-NonCommercial-NoDerivs 4.0 International License (CC BY-NC-ND 4.0), which permits the noncommercial replication and distribution of the article with the strict proviso that no changes or edits are made and the original work is properly cited (including links to both the formal publication through the relevant DOI and the license). See: https://creativecommons.org/licenses/by-nc-nd/4.0/.

\section{References}

1. Varacallo M, Luo TD, Johanson NA. Total Hip
Arthroplasty (THA) Techniques. StatPearls. Treasure Island (FL), 2020.

2. Deak N, Varacallo M. Hip Precautions. StatPearls. Treasure Island (FL), 2020.

3. Kurtz SM, Lau E, Ong K, et al. Future young patient demand for primary and revision joint replacement: national projections from 2010 to 2030. Clin Orthop Relat Res 2009;467:2606-12.

4. Lavernia C, D'Apuzzo M, Hernandez V, et al. Thigh pain in primary total hip arthroplasty: the effects of elastic moduli. J Arthroplasty 2004;19:10-6.

5. Sas A, Pellikaan P, Kolk S, et al. Effect of anatomical variability on stress-shielding induced by short calcarguided stems: Automated finite element analysis of 90 femora. J Orthop Res 2019;37:681-8.

6. King SW, Lamb JN, Cage ES, et al. Periprosthetic femoral fractures following total hip and total knee arthroplasty. Maturitas 2018;117:1-5.

7. Crawford DA, Adams JB, Morris MJ, et al. Distal femoral cortical hypertrophy not associated with thigh pain using a short stem femoral implant. Hip Int 2020:1120700020913872.

8. Brown TE, Larson B, Shen F, et al. Thigh pain after cementless total hip arthroplasty: evaluation and management. J Am Acad Orthop Surg 2002;10:385-92.

9. Jo WL, Lee YK, Ha YC, et al. Frequency, Developing Time, Intensity, Duration, and Functional Score of Thigh Pain After Cementless Total Hip Arthroplasty. J Arthroplasty 2016;31:1279-82.

10. Gallart X, Riba J, Fernandez-Valencia JA, et al. Hip prostheses in young adults. Surface prostheses and short-stem prostheses. Rev Esp Cir Ortop Traumatol 2018;62:142-52.

11. Choy GG, Roe JA, Whitehouse SL, et al. Exeter short stems compared with standard length Exeter stems: experience from the Australian Orthopaedic Association National Joint Replacement Registry. J Arthroplasty 2013;28:103-9.e1.

12. Stulberg SD, Patel RM. The short stem: promises and pitfalls. Bone Joint J 2013;95-B:57-62.

13. Castelli CC, Rizzi L. Short stems in total hip replacement: current status and future. Hip Int 2014;24 Suppl 10:S25-8.

14. Leali A, Fetto J, Insler H, et al. The effect of a lateral flare feature on implant stability. Int Orthop 2002;26:166-9.

15. Dabirrahmani D, Hogg M, Kohan L, et al. Primary and long-term stability of a short-stem hip implant. Proc Inst Mech Eng H 2010;224:1109-19.

16. Ferguson RJ, Broomfield JA, Malak TT, et al. Primary 
stability of a short bone-conserving femoral stem: a twoyear randomized controlled trial using radiostereometric analysis. Bone Joint J 2018;100-B:1148-56.

17. Meyer JS, Freitag T, Reichel H, et al. Periprosthetic Bone Mineral Density Changes After Implantation of a Curved Bone Preserving Hip Stem Compared to a Standard Length Straight Stem: 5-Yr Results of a Prospective, Randomized DXA-Analysis. J Clin Densitom 2019;22:96-103.

18. Samy AM, El-Tantawy A. Stem length in primary cementless total hip arthroplasty: Does it make a difference in bone remodeling? Eur J Orthop Surg Traumatol 2019;29:1235-42.

19. Gielis WP, van Oldenrijk J, Ten Cate N, et al. Increased Persistent Mid-Thigh Pain After Short-Stem Compared With Wedge-Shaped Straight-Stem Uncemented Total Hip Arthroplasty at Medium-Term Follow-Up: A Randomized Double-Blinded Cross-Sectional Study. J Arthroplasty 2019;34:912-9.

20. Liang HD, Yang WY, Pan JK, et al. Are short-stem prostheses superior to conventional stem prostheses in primary total hip arthroplasty? A systematic review and meta-analysis of randomised controlled trials. BMJ Open 2018;8:e021649.

21. Huo SC, Wang F, Dong LJ, et al. Short-stem prostheses in primary total hip arthroplasty: A meta-analysis of randomized controlled trials. Medicine (Baltimore) 2016;95:e5215.

22. Arya S, Schwartz TA, Ghaferi AA. Practical Guide to Meta-analysis. JAMA Surg 2020;155:430-1.

23. Moher D, Shamseer L, Clarke M, et al. Preferred reporting items for systematic review and meta-analysis protocols (PRISMA-P) 2015 statement. Systematic reviews 2015;4:1.

24. Higgins JP, Altman DG, Gotzsche PC, et al. The Cochrane Collaboration's tool for assessing risk of bias in randomised trials. BMJ 2011;343:d5928.

25. Sluimer JC, Hoefnagels NH, Emans PJ, et al. Comparison of two hydroxyapatite-coated femoral stems: clinical, functional, and bone densitometry evaluation of patients randomized to a regular or modified hydroxyapatitecoated stem aimed at proximal fixation. J Arthroplasty 2006;21:344-52.

26. Carlsson LV, Albrektsson BE, Albrektsson BG, et al. Stepwise introduction of a bone-conserving osseointegrated hip arthroplasty using RSA and a randomized study: I. Preliminary investigations--52 patients followed for 3 years. Acta Orthop 2006;77:549-58.
27. Kim YH, Choi Y, Kim JS. Comparison of bone mineral density changes around short, metaphyseal-fitting, and conventional cementless anatomical femoral components. J Arthroplasty 2011;26:931-40.e1.

28. Kim $\mathrm{YH}, \mathrm{Oh} \mathrm{JH}$. A comparison of a conventional versus a short, anatomical metaphyseal-fitting cementless femoral stem in the treatment of patients with a fracture of the femoral neck. J Bone Joint Surg Br 2012;94:774-81.

29. von Roth P, Perka C, Mayr HO, et al. Reproducibility of femoral offset following short stem and straight stem total hip arthroplasty. Orthopedics 2014;37:e678-84.

30. Salemyr M, Muren O, Ahl T, et al. Lower periprosthetic bone loss and good fixation of an ultra-short stem compared to a conventional stem in uncemented total hip arthroplasty. Acta Orthop 2015;86:659-66.

31. McCalden RW, Korczak A, Somerville L, et al. A randomised trial comparing a short and a standard-length metaphyseal engaging cementless femoral stem using radiostereometric analysis. Bone Joint J 2015;97-B:595-602.

32. Freitag T, Hein MA, Wernerus D, et al. Bone remodelling after femoral short stem implantation in total hip arthroplasty: 1-year results from a randomized DEXA study. Arch Orthop Trauma Surg 2016;136:125-30.

33. Kim YH, Park JW, Kim JS. Ultrashort versus Conventional Anatomic Cementless Femoral Stems in the Same Patients Younger Than 55 Years. Clin Orthop Relat Res 2016;474:2008-17.

34. Koyano G, Jinno T, Koga D, et al. Comparison of Bone Remodeling Between an Anatomic Short Stem and a Straight Stem in 1-Stage Bilateral Total Hip Arthroplasty. J Arthroplasty 2017;32:594-600.

35. Schilcher J, Ivarsson I, Perlbach R, et al. No Difference in Periprosthetic Bone Loss and Fixation Between a Standard-Length Stem and a Shorter Version in Cementless Total Hip Arthroplasty. A Randomized Controlled Trial. J Arthroplasty 2017;32:1220-6.

36. van Oldenrijk J, Scholtes VAB, van Beers L, et al. Better early functional outcome after short stem total hip arthroplasty? A prospective blinded randomised controlled multicentre trial comparing the Collum Femoris Preserving stem with a Zweymuller straight cementless stem total hip replacement for the treatment of primary osteoarthritis of the hip. BMJ Open 2017;7:e014522.

37. Yan SG, Li D, Yin S, et al. Periprosthetic bone remodeling of short cementless femoral stems in primary total hip arthroplasty: A systematic review and meta-analysis of randomized-controlled trials. Medicine (Baltimore) 2017;96:e8806. 
38. Leali A, Fetto JF. Preservation of femoral bone mass after total hip replacements with a lateral flare stem. Int Orthop 2004;28:151-4.

39. Khanuja HS, Banerjee S, Jain D, et al. Short boneconserving stems in cementless hip arthroplasty. J Bone Joint Surg Am 2014;96:1742-52.

40. Formica M, Cavagnaro L, Basso M, et al. What is the fate of the neck after a collum femoris preserving prosthesis? a nineteen years single center experience. Int Orthop

Cite this article as: Zhang Z, Xing Q, Li J, Jiang Z, Pan Y, $\mathrm{Hu}$ Y, Wang L. A comparison of short-stem prostheses and conventional stem prostheses in primary total hip arthroplasty: a systematic review and meta-analysis of randomized controlled trials. Ann Transl Med 2021;9(3):231. doi: 10.21037/atm-204043
2017;41:1329-35.

41. Liu Z, Hu H, Liu S, et al. Relationships between the femoral neck-preserving ratio and radiologic and clinical outcomes in patients undergoing total-hip arthroplasty with a collum femoris-preserving stem. Medicine (Baltimore) 2019;98:e16926.

42. Zhang X, Hua T, Zhu J, et al. Body compositions differently contribute to BMD in different age and gender: a pilot study by QCT. Arch Osteoporos 2019;14:31. 

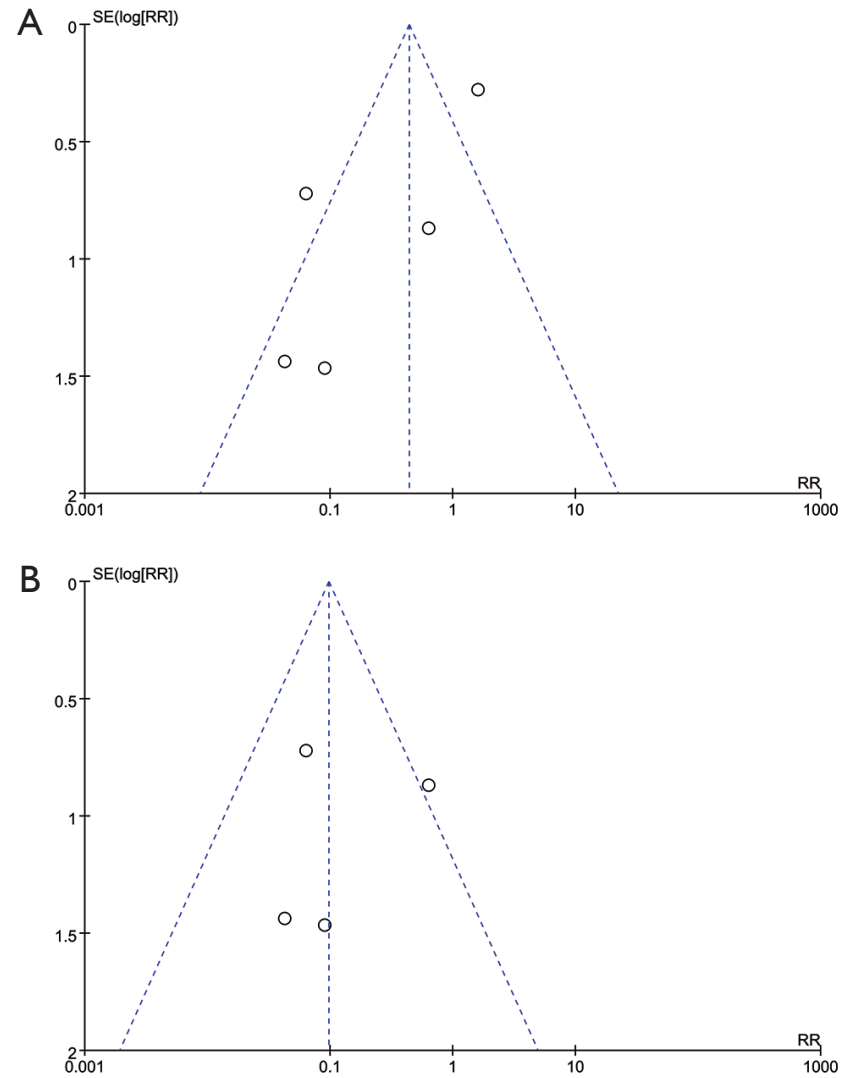

Figure S1 Funnel plot for thigh pain. (A) Including Geilis' RCT; (B) Excluding Geilis' RCT.
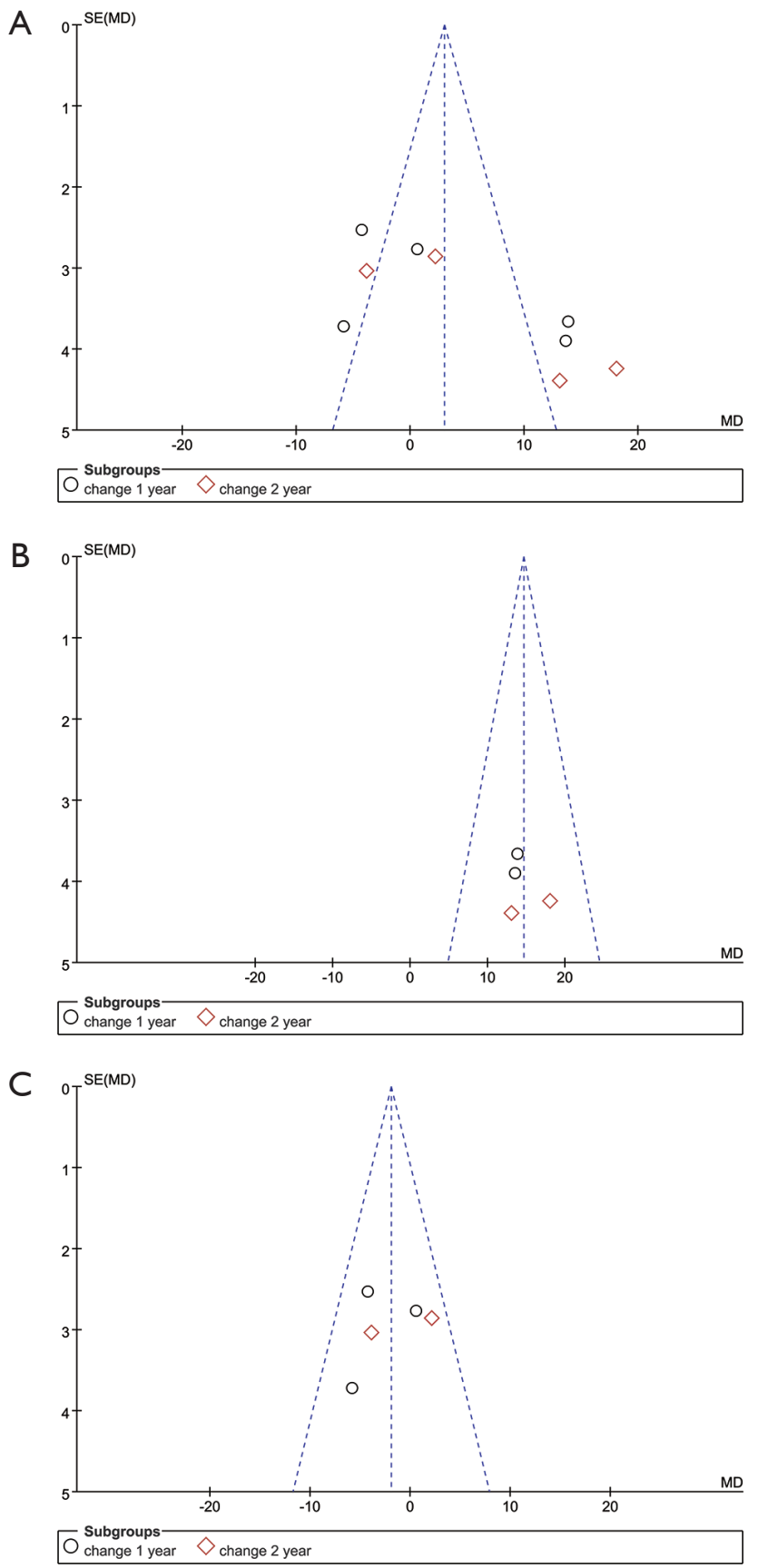

Figure S2 Funnel plot for BMD changes in Gruen zone 1. (A) Including all types of SS; (B) Including SS (I); and (C) Including SS (II). 


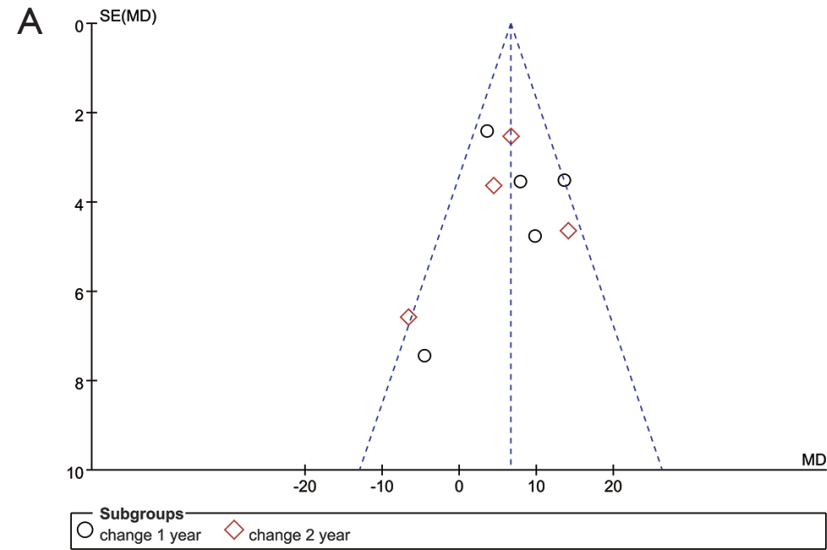

B

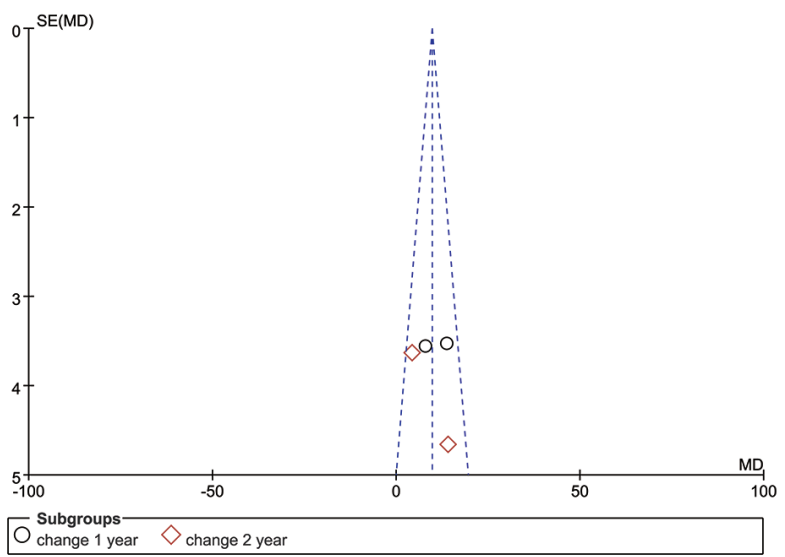

C

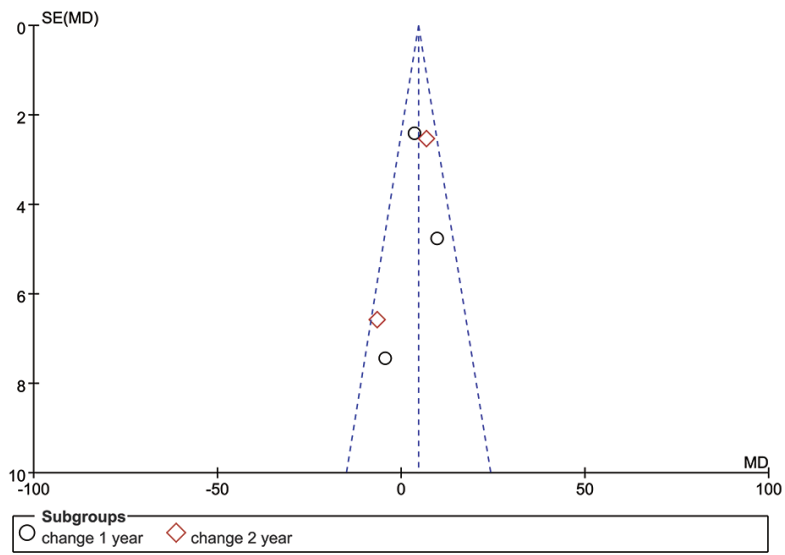

Figure S3 Funnel plot for BMD changes in Gruen zone 7. (A) Including all types of SS; (B) Including SS (I); and (C) Including SS (II).

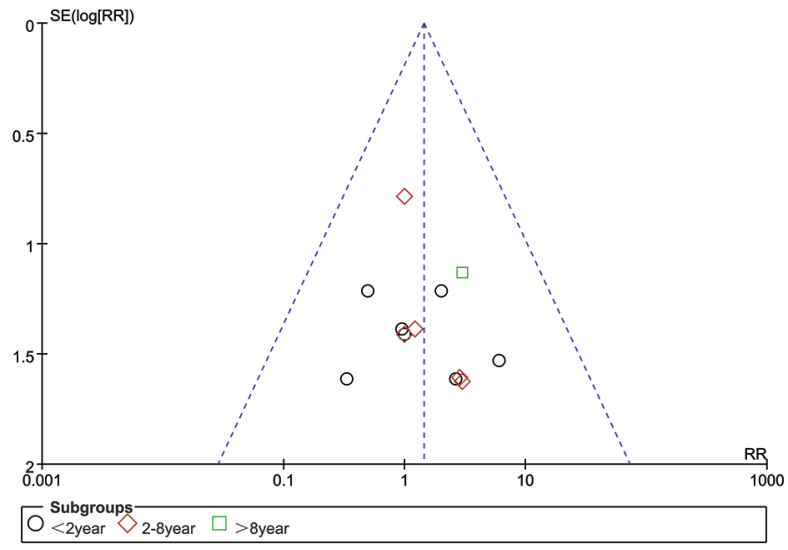

Figure S4 Funnel plot for revision rates.

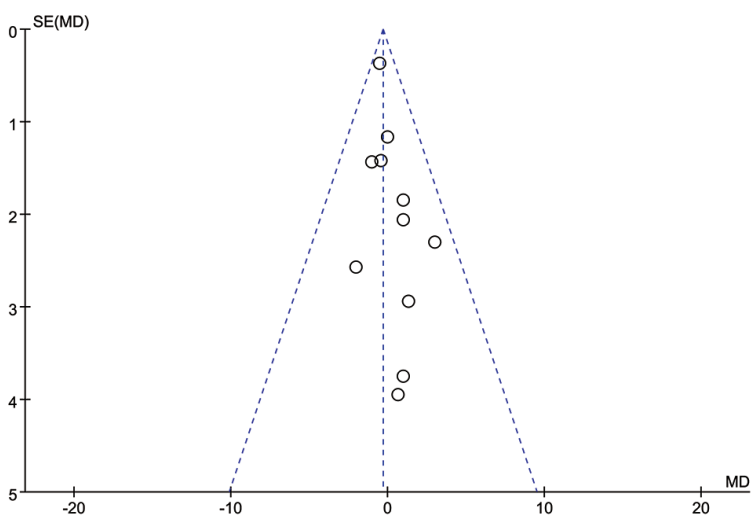

Figure S5 Funnel plot for Harris Hip Scores.

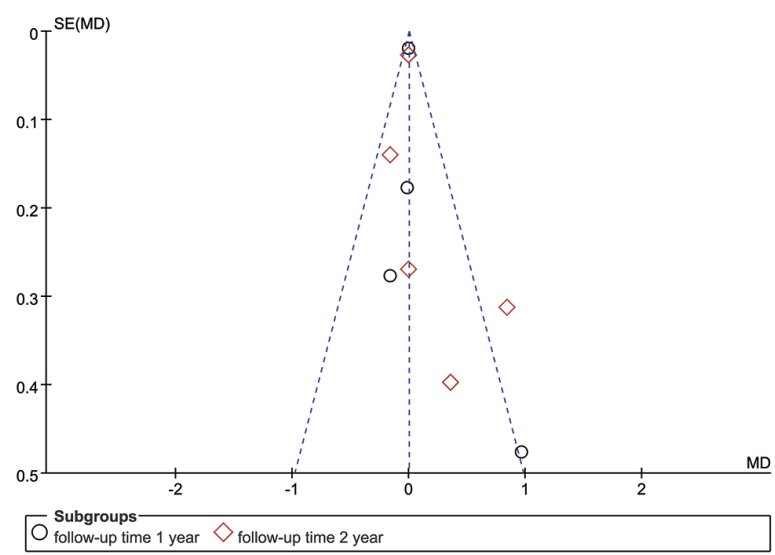

Figure S6 Funnel plot for maximum total point motion. 